\title{
Inventory of Galaxy Properties in Small Scale Structures
}

\author{
L. Tanvuia ${ }^{1}$, B. Kelm ${ }^{2}$, P. Focardi ${ }^{2}$, R. Rampazzo ${ }^{3}$ and W. W. Zeilinger ${ }^{1}$
}

\begin{abstract}
We report on a broad-band $\mathrm{R}$ surface photometric and low resolution spectroscopic study of a set of galaxies located in small groups and pairs of galaxies considered to be in low density environment. Groups span a wide range in density and show different morphological mix. We are analyzing systemic velocities, photometric parameters and spectral properties of the dominant galaxies in the groups. Our aim is to investigate the connection between interaction and induced galaxy activity, from star formation to AGN activity.
\end{abstract}

\section{Introduction}

During the last two decades we learned that interactions could severely alter the properties of a galaxy up to modifying its original morphological class (see reviews of Schweizer 1998, Barnes 1998 and Kennicutt 1998 and references therein). Encounters could then re-direct the evolution of a galaxy. In this picture, the environment plays a key role since it basically governs the type of encounters. A connection should then exist between the local environment and the global properties of galaxies inhabiting it. This link between the environment and the galaxy evolution is still not fully explored for small scale structures in low density environments (LDE), even in the local Universe.

In LDE, galaxy encounters are less frequent than in galaxy clusters and may lead to a merging (Barnes 1998), differently from clusters (Moore et al. 1996). Although galaxies could survive the encounters, depending on the impact parameters, the sudden variation of the potential induces strong modification in their morphological properties (see Schweizer 1998). Star formation episodes could re-juvenate their spectro-photometric properties (see Longhetti et al. 1999 and references therein) as a consequence of the fresh gas refueling. Furthermore, it is still controversial if the interaction plays a dominant role in activating an AGN nucleus or it is only marginally linked to the presence of activity phenomena (see e.g. Kelm, Focardi \& Palumbo 1998 and references therein).

We illustrate here an on-going project for studying small scale structures in LDE analyzing photometric and spectro-photometric data for main members of each group. Our objectives are:

\footnotetext{
${ }^{1}$ Institut für Astronomie, Universität Wien, Türkenschanzstraße 17, A-1180 Wien

${ }^{2}$ Dipartimento di Astronomia, Università di Bologna, Via Ranzani 1, Bologna, Italy

${ }^{3}$ Osservatorio Astronomico di Brera, Via Brera 28, I-20121 Milano, Italy
} 
- to investigate the photometric and structural properties of galaxies in order to perform a comparative analysis with objects resident in dense regions (see Rampazzo et al. 1999) and

- to analyze the possible induced activity, from star formation events to nuclear activity, using a medium resolution spectroscopy and specific models for the interpretation.

\section{The Sample, Observations and Preliminary Results}

The sample is defined using an algorithm which computes the local and the environment space density for each single galaxy using the redshift information. The algorithm (for a detailed description see Kelm, Focardi \& Palumbo, this meeting) produces samples of close associations (e.g. isolated pairs of galaxies compact groups, etc.) having similar environmental characteristics (e.g. in LDE, medium or high density environments).

Long-slit spectra were acquired at ESO $1.52 \mathrm{~m}$ telescope equipped with a Boller \& Chivens spectrograph using a FA 2048L UV Coated CCD (ESO CCD $\# 15)$, in the range $3500 \leq \lambda \leq 11000 \AA$ with a dispersion of $3.7 \AA \mathrm{pix}^{-1}$. Imaging was carried out with the $0.91 \mathrm{~m}$ ESO-Dutch telescope. Bessel $R$ band images were obtained with a SIT $512 \times 512$ pixel CCD detector (ESO CCD \#33) with a scale of 0.442 arcsec $\mathrm{pix}^{-1}$ yielding a field of view of $3^{\prime} .8 \times 3^{\prime} .8$. Present observations consist of 23 multiplets of galaxies. Spectra and images were calibrated using standard procedures in IRAF and MIDAS. Systemic velocities were derived both from absorption (FCORR) and emission lines. The analysis of emission line ratios, looking for induced activity, is in progress.

At the present stage of the reduction, we obtained an independent check of the systemic velocities of the multiplet members necessary to test our selection and the density of the environment (Table 1). The systemic velocity of a consistent fraction of objects shows a remarkable difference with the values reported in the literature. Excluding these objects, we found an average systemic velocity difference (ours - literature) of $57 \mathrm{~km} \mathrm{~s}^{-1}$ with a standard deviation of 123 $\mathrm{km} \mathrm{s}^{-1}$.

\section{References}

Barnes, J. 1998, in Interaction and Induced Star Formation, Saas-Fee Advanced Course 26, Lecture Notes 1996, p.275

Schweizer, F. 1998, in Interaction and Induced Star Formation, Saas-Fee Advanced Course 26, Lecture Notes 1996, p. 105

Kelm, B., Focardi, P. and Palumbo G. G. C. 1998, A\&A, 335, 912

Kennicutt, R. 1998, in Interaction and Induced Star Formation, Saas-Fee Advanced Course 26, Lecture Notes 1996, p.1

Longhetti, M., Bressan, A., Chiosi, C. and Rampazzo, R. 1999, A\&A, 345, 519

Moore, B., Katz, N., Lake, G., Dressler, A. and Oemler A. Jr. 1996, Nature, 379,613 
Table 1. Salient spectroscopic results and properties of multiplets.

\begin{tabular}{|c|c|c|c|c|c|c|c|}
\hline $\begin{array}{l}\text { Group } \\
\text { ident. }\end{array}$ & $\begin{array}{c}\text { RA } \\
(2000)\end{array}$ & $\begin{array}{r}\text { Dec } \\
(2000)\end{array}$ & $\mathrm{T}$ & $\begin{array}{r}\mathrm{V}_{h e l} \\
\mathrm{kms}^{-1} \\
\text { abs. }\end{array}$ & $\begin{array}{c}\mathrm{V}_{h e l} \\
\mathrm{kms}^{-1} \\
\text { emis. }\end{array}$ & $\begin{array}{c}\text { objects } \\
\text { within } \\
\langle 1 \mathrm{Mpc}\rangle\end{array}$ & Notes $^{\alpha}$ \\
\hline$\# 1 \mathrm{a}$ & 171508.8 & 082722 & $\mathrm{E}$ & $10001 \pm 11$ & & $1 \mathrm{C}$ & EL, opt. align. \\
\hline $\mathrm{b}$ & 171512.0 & 082442 & $\mathrm{~S}$ & $6444 \pm 10$ & $6299 \pm 6$ & & \\
\hline$\# 2 a$ & 171725.2 & 074143 & S0 & $6730 \pm 11$ & & $3 \mathrm{C}+4 \mathrm{pC}$ & Group \\
\hline $\mathrm{b}$ & 171733.4 & 073943 & S0 & $6367 \pm 13$ & & & \\
\hline$\# 3 \mathrm{a}$ & 173150.0 & 062854 & $\mathrm{E} / \mathrm{SO}$ & $6846 \pm 13$ & & $6 \mathrm{C}+8 \mathrm{pC}$ & Group \\
\hline $\mathrm{b}$ & 173150.0 & 062954 & $\mathrm{E}$ & $6686 \pm 15$ & & & \\
\hline c & 173156.7 & 062803 & $\mathrm{~S}$ & & $6631 \pm 174$ & & \\
\hline d & 173156.0 & 063154 & $\mathrm{~S}$ & & $6586 \pm 33$ & & \\
\hline$\# 7 \mathrm{a}$ & 205023.6 & -524438 & So & $13224 \pm 6$ & & $24 \mathrm{C}$ & cluster outskirts \\
\hline $\mathrm{b}$ & 205028.6 & -524457 & $\mathrm{~S}$ & & $14450 \pm 66$ & & \\
\hline c & 205032.7 & -524619 & $\mathrm{E}$ & $6935 \pm 152$ & & & \\
\hline$\# 8 \mathrm{a}$ & 205048.0 & -525717 & $\mathrm{E}$ & $12334 \pm 13$ & & $23 \mathrm{C}$ & cluster outskirts \\
\hline$" \mathrm{~b}$ & $2050 \quad 48.9$ & -525604 & $\mathrm{~S}$ & & $12745 \pm 42$ & & \\
\hline c & 205053.2 & -525435 & So & & $12501 \pm 43$ & & \\
\hline$\# 9 \mathrm{a}$ & 205106.3 & -523256 & So & $13479 \pm 7$ & & & opt. align. \\
\hline $\mathrm{b}$ & 204727.8 & -524411 & So & & $14789 \pm 81$ & & \\
\hline$\# 10 \mathbf{a}$ & 205104.4 & -524458 & & & $14362 \pm 31$ & $3 C+3 p C$ & \\
\hline & 205107.1 & -524330 & & & $14825 \pm 37$ & & \\
\hline$\# 12 \mathbf{a}$ & 205208.8 & -530545 & So & $13684 \pm 9$ & & $30 \mathrm{C}$ & opt. align. \\
\hline & 205211.5 & -530524 & So/S & & $9152 \pm 47$ & & \\
\hline$\# 16 \mathbf{a}$ & 205947.3 & -015312 & $\mathrm{I}$ & & $5767 \pm 47$ & isolated & $P$ \\
\hline & 205948.4 & -015223 & $\mathrm{~S}$ pec & & $5770 \pm 7$ & & \\
\hline$\# 17 \mathbf{a}$ & 214514.5 & -200028 & $\mathbf{E}$ & $17183 \pm 6$ & & $1 C+4 p C$ & $\mathbf{P}$ \\
\hline & 214515.5 & -195940 & $\mathbf{E}$ & $17563 \pm 11$ & & & \\
\hline \#18a & 215404.1 & -160928 & $\mathbf{S}$ & & $11083 \pm 2$ & $1 \mathrm{pC}$ & $\mathbf{P}$ \\
\hline & 215405.3 & -160932 & $S$ & & $11066 \pm 6$ & & \\
\hline$\# 19 a$ & $\begin{array}{lll}22 & 01 & 01.7\end{array}$ & 080634 & $\mathrm{E} / \mathrm{SO}$ & $8474 \pm 11$ & & $1 \mathrm{C}+8 \mathrm{pC}$ & $\mathrm{P}$ \\
\hline & 220053.0 & 080230 & $\mathrm{~S}$ & & $8578 \pm 6$ & & \\
\hline$\# 26 \mathrm{a}$ & 225030.4 & -191434 & $\mathrm{E}$ & $20531 \pm 14$ & & $3 \mathrm{pC}$ & opt. align. \\
\hline $\mathrm{b}$ & 225037.6 & -191336 & So pec & & $21940 \pm 37$ & & \\
\hline$\# 27 a$ & 225851.1 & -073456 & $\mathbf{E}$ & $23136 \pm 7$ & & $1 \mathrm{pC}$ & $\mathbf{P}$ \\
\hline b & 225854.5 & -073608 & $\mathrm{E}$ & $23247 \pm 5$ & & & \\
\hline $\mathrm{c}$ & 225855.5 & -073654 & So/E & $9716 \pm 7$ & & & \\
\hline$\# 29 a$ & 000234.8 & -034240 & $\mathbf{S}$ & & $6448 \pm 30$ & $1 C+3 p C$ & $\mathbf{P}$ \\
\hline & 000238.5 & -033751 & $\mathrm{SO} / \mathrm{S}$ & & $6242 \pm 11$ & & \\
\hline \#31a & $\begin{array}{lll}00 & 03 & 22.3\end{array}$ & -104614 & So & $8861 \pm 11$ & & isolated & $\mathbf{P}$ \\
\hline & $00 \quad 03 \quad 32.1$ & -104440 & $\mathbf{S}$ & $8788 \pm 19$ & & & \\
\hline$\# 32 a$ & 001611.5 & 022642 & $\mathrm{~S}$ & & $17527 \pm 1$ & $2 \mathrm{pC}$ & \\
\hline & $\begin{array}{lll}00 & 16 & 13.9\end{array}$ & 022716 & $\mathrm{~S}$ & & $17491 \pm 6$ & & \\
\hline$\# 35$ a & 003022.0 & 065736 & E/SO & $18010 \pm 13$ & & $2 \mathrm{pC}$ & $P+2$ bkg obj. \\
\hline $\mathrm{b}$ & 003028.7 & 065834 & $\mathrm{~S}$ & & $17366 \pm 25$ & & \\
\hline $\mathrm{C}$ & 003034.7 & 065634 & $\mathrm{~S} 0 / \mathrm{E}$ & & $19717 \pm 134$ & & \\
\hline$\# 36 \mathbf{a}$ & $\begin{array}{lll}00 & 4147.0\end{array}$ & $08 \quad 22 \quad 24$ & $\mathbf{E}$ & $12714 \pm 6$ & & $3 \mathrm{pC}$ & $\mathbf{P}$ \\
\hline & 004130.0 & 082124 & SB & $13000 \pm 11$ & $12872 \pm 19$ & & \\
\hline \#56а & 015126.0 & -082453 & pec & & $15445 \pm 4$ & $2 \mathrm{pC}$ & opt. align. \\
\hline & $\begin{array}{lll}01 & 5134.0\end{array}$ & -082356 & SB & & $5389 \pm 2$ & & \\
\hline$\# 58 \mathrm{a}$ & $\begin{array}{llll}02 & 02 & 17.3\end{array}$ & -010740 & $E$ & $12707 \pm 13$ & & $1 p C$ & $\mathrm{P}$ \\
\hline & $\begin{array}{lll}02 & 02 & 20.3\end{array}$ & -010638 & $\mathrm{E} / \mathrm{SO}$ & $12920 \pm 12$ & & & \\
\hline \#63a & 023734.8 & $\begin{array}{lll}-11 & 0134\end{array}$ & $\mathrm{~s}$ & $4588 \pm 11$ & $4694 \pm 15$ & $5 \mathrm{C}+1 \mathrm{pC}$ & \\
\hline & 023738.9 & -110020 & $\mathbf{E}$ & $4754 \pm 17$ & & & \\
\hline \#65a & 024358.5 & 052619 & $\mathbf{E}$ & $7046 \pm 14$ & & $2 \mathrm{pC}$ & \\
\hline $\mathrm{b}$ & $0244 \quad 05.0$ & $\begin{array}{lll}05 & 26 & 12\end{array}$ & $\mathrm{~S}$ & & $7250 \pm 7$ & & \\
\hline
\end{tabular}

${ }^{a}$ Notes: EL, indicating the presence of emission line, is reported only for early-type galaxies. $\mathrm{P}=$ pair; $\mathrm{C}=$ Companion (the redshift is known); $\mathrm{pC}=$ possible companion the redshift is not known. We consider in LDE galaxies for which within $1 \mathrm{Mpc}$ there are less than 5 companions. 


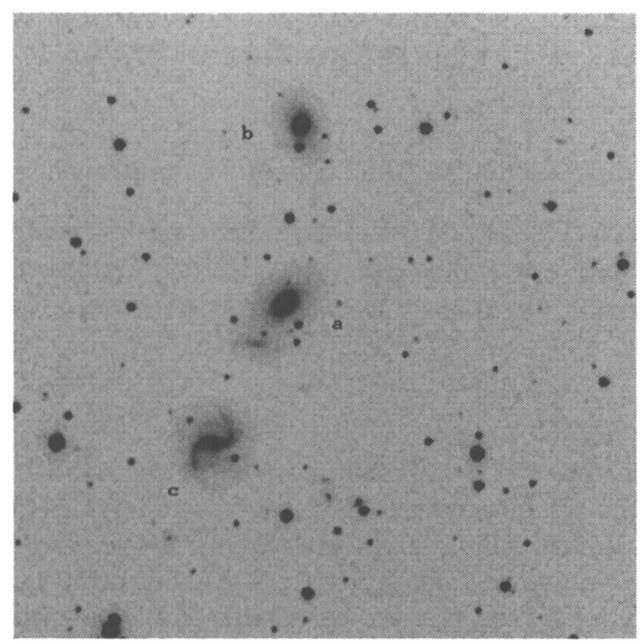

Figure 1. R-band image of three members in the multiplet \#3. None of the early-type galaxies show fine structure.

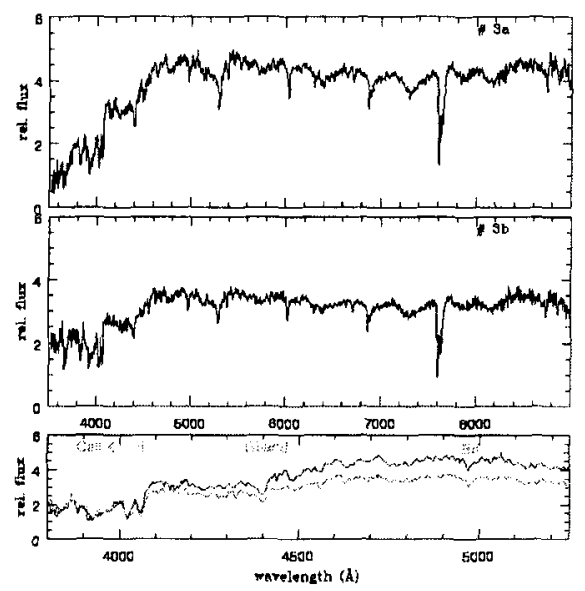

Figure 2. Composition of early-type galaxies spectra in the multiplet $\# 3$. 
Rampazzo, R., D'Onofrio, M., Bonfanti, P. Longhetti, M. and Reduzzi, L. 1999, Ap Letters \& Communications, in press. 